\section{Basal Forebrain}

Randall E. Merchant

Department of Neurosurgery, Virginia

Commonwealth University, Richmond, VA, USA

\section{Definition}

The basal forebrain is a collection of nuclei and tracts that lie near the bottom and front of the brain. It includes the nucleus basalis, diagonal band of Broca, and medial septal nuclei. This area's neurons are major producers of acetylcholine which is then distributed throughout the brain and most importantly to the cerebral cortex and amygdala. The basal forebrain is most commonly damaged by an aneurysm of the anterior communicating artery. When this occurs, there is a reduction in the amount of acetylcholine in the brain, leading to impaired learning, amnesia, and confabulation. A decrease in cholinergic output by neurons of the basal forebrain is also known to occur in cases of Alzheimer's disease and senile dementia.

\section{Cross-References}

Anterior Communicating Artery 